\title{
Violencia escolar en la ciudad de Bluefields, RACCS Nicaragua
}

\author{
Eva Hodgson Suárez ${ }^{1}$ \\ Verónica Donaire Mena ${ }^{2}$ \\ Dora Ibarra Ramírez ${ }^{3}$
}

\section{Resumen}

Este estudio ha analizado las manifestaciones de Acoso Escolar (Bullying), causas y alteraciones psicológicas que ocasiona en estudiantes de Educación Secundaria de la ciudad de Bluefields. Es de tipo cuantitativo y cualitativo; la encuesta fue aplicada a 263 estudiantes de un universo de 536; estos datos fueron procesados a través del SPSS 0.17. También se desarrollaron dos grupos focales con la participación de 14 estudiantes y entrevistas individuales a 15 docentes. La información brindada fue analizada con las técnicas de análisis de contenido y discurso.

Los resultados han revelado que los centros educativos de la ciudad de Bluefields no están exentos a la problemática del acoso escolar. Las manifestaciones de este fenómeno incluyen agresiones verbales, psicológicas, físicas y en menor escala las de índole sexual. Estas acciones desencadenan alteraciones emocionales en las víctimas de acoso, tales como el aislamiento social, baja autoestima, temor e ideas suicidas, esto también disminuye su rendimiento escolar. Las principales causas del acoso escolar es la violencia intrafamiliar, y la discriminación por pertenecer a grupos étnicos minoritarios. En cuanto a las estrategias de afrontamientos más utilizadas por los estudiantes para manejar situaciones de acoso escolar se refieren las centradas en la resolución y las estrategias de evitación.

Palabras Clave: acoso escolar; alteraciones psicológicas; estrategias de afrontamiento.

\section{Summary}

This study has analyzed the manifestations of Bullying, causes and psychological alterations that it causes in the students of Secondary Education of the city of Bluefields. It is based on a quantitative and qualitative approach. The survey was applied to 263 students from a universe of 536 , after which the data's were processed through SPSS 0.17. Two focus groups was also developed with the participation of 14 students, as

\footnotetext{
Master académica en Salud Ocupacional. Psicóloga. Docente en URACCAN. Correo electrónico: eva.hodgson@uraccan.edu.ni Licenciada en Psicología en Contextos Multiculturales. Docente en URACCAN. Correo electrónico: verodonaire@yahoo.es Licenciada en Psicología en Contextos Multiculturales. Centro de atención psicosocial Siuna. Correo electrónico: yanin_ibarra@ yahoo.com
} 


\section{EDUCACIÓN}

well as individual interviews with 15 teachers. The information provided was analyzed through the techniques of analysis of content and speech.

The results have revealed that the schools in the city of Bluefields are not exempt to the problem of bullying. The manifestations of this phenomenon include verbal, psychological and physical aggressions, and in a lesser degree, sexual aggressions. These actions causes emotional alterations to the victims of harassment, such as social isolation, low self-esteem, fear and suicidal ideas, and it also weakens their school performance. The main causes of bullying are domestic violence and discrimination due to their belonging to minority ethnic groups. Regarding the strategies of confrontations that are most used by the students to handle situations of school bullying, they refer to those focused on the resolution and strategies of avoidance.

Keywords: School bullying; Psychological alterations; strategies of confrontation.

\section{Introducción}

La violencia en sus múltiples manifestaciones es un fenómeno presente en las instituciones de educación formal. Centros de desarrollo Infantil, escuelas y colegios a diario enfrentan situaciones de acoso y hostigamiento generado entre estudiantes y/o maestros. Estos conflictos crean un ambiente que obstaculizan el desarrollo de los procesos de enseñanza aprendizaje. Este estudio busca entender las causas del acoso escolar, así como las repercusiones que genera en la psiquis de estudiantes y docentes.

Mediante la implementación de técnicas de indagación cuantitativas y cualitativas, en el 2012, se analizaron dos centros Educación Secundaria ubicados en la ciudad de Bluefields. Estudiantes y docentes afirman que, en estos sitios diseñados para generar conocimientos, saberes y valores, a diario se experimentan situaciones de violencia de índole física, psicológica y sexual. Aseveran que estos comportamientos son un reflejo directo de la violencia intrafamiliar a la cual son sometidos los estudiantes, así como los docentes. Sin embargo, en un contexto multicultural en el que convergen pueblos afrodescendientes, indígenas y mestizos, también aseguran que los conflictos interétnicos, producen discriminación racial, siendo esta otra causa de violencia.

Las principales afectaciones psicológicas que desencadenan la violencia escolar son el aislamiento social, baja autoestima, fobia escolar e ideas suicidas, los cuales repercuten negativamente en el rendimiento escolar de los estudiantes. Docentes externan profunda preocupación dado a que los procesos educativos se ven fragmentados por la violencia escolar. Además, carecen de las estrategias para prevenir y/o evitar la violencia en las escuelas. Las víctimas directas de violencia escolar indican que sus estrategias de afrontamiento son las centradas en la resolución y las estrategias de evitación. Sin embargo, admiten que estas no son suficiente y generalmente ameritan la intervención de profesores, madres y padres de familias. 
Estos resultados permiten a las autoridades regionales, educativas, docentes, madres y padres de familia, conocer la magnitud de la violencia escolar. A reconocer que este un asunto público que requiere un abordaje holístico. Así mismo, contribuye al establecimiento de centros escolares con ambientes saludables que propicien el desarrollo integral de cada uno de los miembros de la comunidad educativa. En términos académicos este estudio contribuye con la generación de nuevo conocimientos sobre la violencia escolar en una población de la cual poco se ha indagado.

\section{Revisión de literatura}

\section{Generalidades sobre acoso escolar}

El psicólogo escandinavo Olweus, D. (1978, 1993, 1998), define el acoso escolar como la conducta de persecución física o psicológica impuesta por los agresores a sus víctimas. El objetivo de la práctica es intimidar, opacar, reducir, someter o debilitar emocional e intelectualmente a la víctima, con el fin de obtener algún resultado favorable para quienes acosan, satisfaciendo una necesidad imperiosa de dominar, someter, agredir y destruir, estas son características que presentan los acosadores como un patrón predominante de relación social con los demás.

El acoso escolar, también denominado hostigamiento escolar o bullying, se caracteriza por la presencia de agresiones físicas, psicológicas y de índole sexual. Según Rodríguez, D. F.; Gutiérrez, H.C.; Herrero, F. J.; Albuerna, F.; Cuesta, M.; Hernández E.; Gómez, C, P. (2004), este comportamiento genera consecuencias tanto a las víctimas como a victimarios y victimarias. Consecuentemente la sociedad en general está sumergida en este fenómeno.

\section{Causas del acoso escolar}

Oñederra, J. (2008), indica que existen ciertas patologías infantiles que pueden estar relacionadas con la agresividad: niñas y niños con dificultades para el autocontrol, con baja tolerancia a la frustración, trastorno por déficit de atención e hiperactividad (TDAH), niñas y niños de voluntad firme son susceptibles a ejercer violencia sus pares.

Olweus, D. (1998), retomando la teoría del aprendizaje por observación o modelado postulada por Bandura, A. (1977), expone que la principal causa de Acoso Escolar es la violencia intrafamiliar que experimentan los estudiantes. mediante un proceso de aprendizaje de observación e imitación, puede resultar como victimario, o a presentar mayor vulnerabilidad y convertirse en víctima.

Autores como Olweus, D. (1998), Benítez, J. y Justicia F. (2006), coinciden al explicar que la principal causa del acoso escolar son los patrones de crianza implementados por madres y padres de familia. También señala que niñas, niños y adolescentes con 
falta de afecto y cuidado en sus hogares, tienden a desarrollar un comportamiento de dominio sobre otras personas.

Palomero, J. y Fernández, M. (2012) indican que, en los adolescentes, debido a la fase de rebeldía y reproducción de la conducta agresiva como un modelo de masculinidad, les puede conllevar a ejercer acoso. Rojas, M. (1995) y López, J. (2000) explican que la cultura machista, es un factor predominante, considerando que las agresiones físicas la realizan principalmente los varones. Kuntsche E, (2006), también indica la influencia de los medios de comunicación como una fuente de aprendizaje de conductas violentas, las cuales niñas, niños y adolescentes reproducen en sus escuelas.

\section{Consecuencias del acoso escolar}

Los efectos negativos del acoso escolar, lo experimentan tanto la víctima, victimario y testigos: La víctima tiende a pensar que es responsable de la intimidación, esto conlleva a la baja autoestima, actitudes de apatía, trastornos emocionales, trastornos psicosomáticos, depresión, ansiedad, pérdida de interés por la escuela, ideas suicidas y suicidio (Oñederra, J. 2008).

Oñederra, J. (2008), denota que, en los agresores, las conductas de acoso pueden hacerse crónicas y convertirse en una manera ilegítima de alcanzar sus objetivos de dominar a otros. Las principales consecuencias en el victimario o victimaria son baja autoestima, falta de empatía problemas psicosomáticos, el bajo rendimiento académico, fracaso escolar, dificultades para el cumplimiento de normas e incidir en conductas antisociales y delictivas, Incapacidad de establecer relaciones sociales positivas, carece de sentimiento de culpabilidad, ira e impulsividad.

Palmero, J. y Fernández, M. (2012), señalan que los testigos corren el riesgo de insensibilizarse ante las agresiones cotidianas y de no reaccionar a las situaciones de injusticia en su entorno trayendo como consecuencia el miedo, sumisión, pérdida de empatía, desensibilización, falta de solidaridad, sentimiento de culpa e interiorización de conductas antisociales y delictivas para obtener deseos.

\section{Estrategias de afrontamiento ante el acoso}

Hunter, S. Mora, M. y Ortega, R. (2004), concluyen que el acoso escolar produce estrés en las victimas, y retoman la formulación teórica propuestas por Lazarus y Folkman (1984) y Roth y Cohen (1986) para el manejo del estrés. Estos señalan que las estrategias de afrontamiento ante situaciones de acoso escolar, pueden estar dirigidas a dos tipos de metas: Las estrategias centradas en la emoción, dirigidas a regular la emoción buscando el equilibrio perdido y por otro lado las estrategias centradas en la resolución, es decir tratar de entender y resolver el problema mediante la implementación de diversas acciones. 


\section{Materiales y métodos}

El estudio es de carácter cuantitativo y cualitativo, dirigido a explorar los conocimientos que poseen estudiantes y docentes acerca del acoso escolar, identificar las causas que lo generan, las afectaciones psicológicas que desencadenan y las estrategias de afrontamiento que emplean para solucionar o prevenir esta problemática. El ámbito de estudio fueron dos Centros Escolares de Secundaria ubicados en la ciudad de Bluefields (Región Autónoma Costa Caribe Sur de Nicaragua). Las unidades de análisis de los estudiantes que cursan el ciclo Básico de Secundaria (I, II y III año).

En el componente cuantitativo, para la recolección de los datos se aplicó una guía de preguntas cerrada elaborada por las autoras del estudio, aplicada a una muestra aleatoria de 263 estudiantes. Para la recopilación de los datos cualitativos se realizaron dos grupos focales con la participación de 14 estudiantes. También se desarrollaron entrevistas individuales con 15 docentes que laboran en los centros.

En cuanto a las consideraciones éticas tomadas para el desarrollo de esta investigación, se aseguró a los participantes el anonimato y manejo confidencial de la información brindada, también se obtuvo el consentimiento libre previo e informado por escrito tanto de las autoridades escolares y de estudiantes, así como del claustro de profesores. Para la solución de posibles sesgos, se realizó una prueba piloto al cuestionario aplicado, así mismo al momento del llenado se aclararon las preguntas y dudas que manifestaron los participantes, asegurando por ende el entendimiento de las preguntas.

El análisis de los datos cuantitativos fue con el programa estadístico para las ciencias sociales (SPSS versión o.17). La información de los grupos focales y entrevistas se manejó a través de análisis de contenido y del discurso, estos resultados se presentan en cita textual.

\section{Resultados y discusión}

\section{Características socio demográficas}

El 55.5\% (146) del grupo de estudiantes encuestados pertenecen al sexo femenino y el 44.5 (117) al masculino. Se encontró que el rango general de edades oscila entre 11 a 19 años. El mayor porcentaje (58\%) se concentra en las edades entre 14-16 años, la media calculada fueron 11 y 13 años. En cuanto a la distribución étnica de estudiantes, el grupo predominante fue la etnia creole con el 57.8\% (152), mestiza con el 38\%, miskita $2.3 \%$ y $1.9 \%$ garífuna. El nivel de escolaridad que presenta mayor predominio en la población es primer año de secundaria con un porcentaje de $51.7 \%$, con el $38.8 \%$ segundo año, y por último el tercer año de secundaria con el 9.5\%. 


\section{EDUCACIÓN}

En los grupos focales desarrollados, se contó con la participación de 14 estudiantes, entre los rangos de edad de 14-18 años, con presencia de ambos sexos y pertenecientes a las etnias creole, mestiza y miskita. En las entrevistas participaron 8 maestros y 7 maestras, el rango de sus edades oscila entre 21-57 años. la mayoría pertenecen a la etnia mestiza.

Estos datos reflejan que, en estos dos centros escolares, se atienden principalmente a estudiantes pertenecientes a los grupos étnicos minoritarios; sin embargo, el claustro de docentes (78\% aproximadamente) son mestizos. Esta situación genera varios cuestionamientos en relación a la preparación de profesoras y profesores en el manejo de pedagogías para ambientes multiculturales, así como el dominio de las lenguas propias de los estudiantes de manera que se les permita expresarse en su lengua materna. En Bluefields, a pesar de la existencia de un marco jurídico (ley de autonomía, ley de lenguas) que aprueba y demanda el uso de las lenguas autóctonas en los procesos de enseñanza aprendizaje, estudiantes pertenecientes a los grupos étnicos minoritarios están obligados a aprender el español, lo cual genera sentimientos de frustración en aquellos estudiantes que presentan dificultades en el dominio e este idioma. Consecuentemente, esta frustración puede desencadenar conductas de agresión por parte de estos estudiantes.

En materia de los conocimientos que poseen los estudiantes sobre la violencia escolar, los resultados revelan que el $87.3 \%$ de los estudiantes señalan que conocen sobre casos de acoso que se presentan en el centro escolar y que esto sucede principalmente entre los estudiantes; sin embargo, el 36.5\% indicó que los docentes también ejercen acoso hacia el estudiantado.

En los grupos focales, estudiantes definieron la violencia escolar con las siguientes palabras:

Es cuando una persona recibe maltratos físicos y verbales, aparte del maltrato, lo amenazan diciéndoles que lo van a esperar afuera o le mandan mensajes con palabras groseras (estudiante del sexo masculino). También es humillar, empujar a otras personas diciéndoles palabras groseras, como decirles que son feos (estudiante del sexo femenino). Para mí es cuando hay abuso físico y psicológico (estudiante del sexo femenino).

En los grupos focales estudiantes narraron situaciones de casos de abuso y violencia en las escuelas, expresaron:

Yo conozco un caso de una vecina que es amiga mía, ella salía de la casa y las chavalas la esperaban, le tapaban los ojos por atrás, le quitaban la mochila, el dinero, le tiraban los libros. Cuando hacían esto estaban vestidas con sus uniformes. La muchachita 
llegaba llorando a sus padres. Ellos les comunicaron a los padres de estas muchachas y a la directora del centro. Este caso lo arreglaron en la Oficina de Derechos de la Niñez y Adolescencia.

Claro aquí hay varios casos, yo conozco dos casos: el primero es que tienen de mandadero a un chavalo de mi año. $\mathrm{Y}$ a otro que lo golpean y lo molestan, le dicen amenazas que si no lo hace les van a pegar o que les van golpear en la cabeza (estudiante del sexo masculino).

El 73.38\% de los estudiantes indican que los actos de acoso que se presentan con mayor frecuencia es de forma verbal, haciendo énfasis en las burlas, ofensas y palabras descalificativas. El 11.3\% señala la práctica de empujones y golpes. 8.37\% hizo referencia a la exclusión y aislamiento de compañeros, y en un menor porcentaje, pero fuertemente llamativo el $7.22 \%$ señaló la presencia de acoso sexual en la escuela.

$\mathrm{Al}$ indagar sobre quiénes son los que ejercen la violencia escolar, estudiantes refirieron que:

El acoso se da de estudiante a estudiante, pero también los estudiantes a profesores, los estudiantes le gritan, les dicen palabras feas, los ofenden y a pesar de que los profesores saben que son ellos los que mandan sé quedan callados (estudiante del sexo masculino).

También refirieron que:

Hay profesores que son racistas, por ejemplo, nos tratan mal, en los exámenes nos cambian los puntajes; pero, también se ha dado racismo de estudiante a profesores a veces no los quieren por ser mestizo (estudiante del sexo femenino). Con estos datos, nuevamente se puede discutir sobre la preparación de los docentes en la implementación de pedagogías adecuadas para el manejo de ambientes multiculturales. Así como analizar con mayor profundad el hecho de que los estudiantes afrodescendientes e indígenas son los que poseen los rendimientos académicos más bajos en las escuelas. Esta situación resulta en un rechazo por parte de estudiantes hacia maestros de la etnia mestiza.

Los docentes también hicieron mención de las diferentes formas de acoso que se presentan en las escuelas:

"Golpes, robos y abuso verbal" "Agresión física y verbal, discriminación, discusiones violentas, pleitos, rechazo grupal y exclusión” "Pegarles a los más pequeños, quitarles el dinero en la hora de receso, darles bromas pesadas" (maestra). 


\section{EDUCACIÓN}

Este estudio sobre acoso escolar en la ciudad de Bluefields revela que la mayoría de estudiantes y docentes conocen este tipo de violencia y las formas en que se manifiestan. También están al tanto de situaciones reales de acoso verbal que se desarrollan en sus escuelas, esto corresponden con las definiciones propuestas por Olweus, D. $(1978,1993,1998)$, quien hace referencia que el acoso escolar es el agravio físico, verbal, psicológico y sexual que se da de un estudiante a otro en repetidas ocasiones. Las expresiones de estudiantes y maestros señalan que en estos centros también se da la violencia verbal por parte de estudiantes hacia docentes.

Contrario a los estudios realizados en otros países, esta investigación desarrollada en la ciudad de Bluefields revela otro elemento causante del hostigamiento escolar, al hacer referencia sobre la existencia de discriminación racial en los centros escolares. Es importante señalar que la mayoría de la población estudiantil beneficiarios de estos centros escolares pertenece al pueblo afrodescendiente creole; sin embargo, la mayoría de docentes que allí laboran son de la etnia mestiza, lo cual contrae otros tipos de limitantes en la comunicación entre profesores y estudiantado, esto podría ser causante de conflictos desencadenados por no lograr una comunicación asertiva. Se hace necesario realizar más indagaciones sobre el tema de la discriminación racial como una causa de acoso escolar, considerando también hasta qué punto inciden las barreras comunicativas en la creación de situaciones violentas y de acoso escolar.

Por otro lado, estudios realizados por Olweus, D. (1993), indican el Acoso Escolar Homofóbico, es decir acoso basado en la orientación sexual de las personas, este último elemento no tuvo mayor significancia en la ciudad de Bluefields, probablemente porque las persona homosexuales y lesbianas no expresan libremente su preferencia sexual, aún así, es pertinente indagar más sobre este aspecto en futuras investigaciones.

En cuanto a las causas del acoso escolar, docentes señalan que estas pueden ser múltiples y complejas, expresaron:

Falta de convivencia, socialización, compañerismo y valores espirituales. Muchos de los estudiantes realizan estas acciones, con el fin de llamar la atención porque en sus hogares carecen de la misma [...] Maltrato en el hogar, falta de comunicación, riñas entre los padres y poco tiempo de calidad entre los padres.

Las aseveraciones de docentes al expresar que los estudiantes reproducen el comportamiento que han aprendido en el núcleo familiar. La mayoría de los agresores sufren de violencia Intrafamiliar. Estos resultados coinciden con los estudios realizado por Olweus, D. (1978, 1993, 1998), y Espinoza, E. (2011); Así mismo concuerdan con Benítez, J. y Justicia, F. (2006). Aquí discutimos que toma relevancia nuevamente aquella aseveración "la educación inicia en el hogar" y por ende queda nuevamente implícita la responsabilidad de los padres y madres en el desarrollo de sus hijas e hijos. 
En cuanto a las afectaciones psicológicas o emocionales que experimentan las victimas del acoso escolar, expresan:

Les bajan la autoestima por el maltrato. Estas amenazas los pueden llevar al suicidio. (estudiante del sexo masculino). Le da miedo cuando ve a las personas que lo agreden (estudiante del sexo femenino), el estudiante se cohíbe socialmente (Docentes). presenta dificultad para relacionarse por temor a ser rechazados; se sienten tristes, lloran, se aíslan y se sienten menos que los demás (estudiante del sexo masculino).

En cuanto al daño psicológico o alteraciones que experimentan las personas ante el acoso escolar o Bullying se identifican la baja autoestima, tristeza, temor a asistir al centro educativo, descenso en el rendimiento escolar, aislamiento, lo cual podría desencadenar ideas suicidas. Podemos observar que el individuo que es víctima de acoso es afectado a nivel general e incluye su entorno familiar, social, relaciones personales e interpersonales, así mismo lo ha señalado Oñederra, S. (2008).

Entre las estrategias para afrontar el acoso sobresalen las estrategias centradas en la resolución de conflictos; los estudiantes señalaron el hablar con las autoridades de su respectivos centros escolares y padres de familia para evitar este tipo de acoso. Y por otro lado las estrategias centradas en la evitación, estudiantes evaden las burlas y ofensas, se apartan. Utilizar las estrategias de evitación para manejar el acoso escolar puede ser más dañino para la víctima.

Ante esto los estudiantes expresaron:

Lo mejor es no quedarse callado y hablar con la directora, maestros/maestras o con sus padres para evitar ser víctima del acoso escolar (estudiante del sexo femenino).

Yo no le pongo mente (estudiante del sexo femenino).

Lo que yo hago es apartarme (estudiante del sexo femenino).

Los docentes expresaron:

Pleitos entre alumnos y palabras obscenas, tuve intervención desapartándolos y dándoles consejos, llamé a los supervisores. (Maestros/as del tiempo completo). Insultos entre dos compañeros, por realizar bromas pesadas. La intervención fue llevarlos a la dirección, llamar a sus padres y a la consejera del centro para charlar con ellos.

La violencia está presente en todos los ámbitos sociales y se manifiestan de diferentes maneras. La ciudad de Bluefields no está exenta del acoso escolar. En materia de las conceptualizaciones, causas y consecuencias los hallazgos encontrados en este estudio coinciden con los resultados de investigaciones desarrolladas en otras zonas 
del país y a nivel internacional. Sin embargo, cabe mencionar que revela la presencia de discriminación racial como una causa del acoso escolar, esta diferencia puede deberse a que en la ciudad de Bluefields así como el resto de la Costa Caribe de Nicaragua, se caracteriza por ser una zona en la que conviven y convergen personas pertenecientes a los pueblos indígenas, afrodescendientes y mestizos (siendo estos últimos el grupo de mayor densidad poblacional), lo que hace de éste un contexto multicultural y plurilingüe, susceptible a las diferencias raciales y discriminación racial. Con esto se introduce entonces, un nuevo elemento que deberá continuar investigándose y discutiendo al abordar el fenómeno del acoso escolar. Lo cual está íntimamente ligado a la preparación que poseen docentes en las pedagogías permita crear ambientes de educativos incluyentes.

\section{Conclusiones}

Este estudio desarrollado con estudiantes y profesores de dos centros de educación a nivel de secundaria en la ciudad de Bluefields, afirma la presencia de acoso escolar. Tanto estudiantes como docentes conocen sobre este tema y sus diversas formas de manifestación.

- Las principales formas de violencia en la escuelas son los insultos, ofensas y palabras descalificativas. Refiriendo también que tanto estudiantes y docentes ejercen este tipo de conducta.

- Los docentes señalan que la principal causa del acoso escolar es la violencia intrafamiliar; sin embargo, estudiantes y profesores han identificado que la discriminación racial es otra causa para la presencia de este tipo de violencia.

- Las alteraciones emocionales que desencadenan las víctimas de acoso escolar sobresalen la inestabilidad emocional, ansiedad, inseguridad, aislamiento, ideas suicidas y en casos extremos el suicidio, todo esto producto del acoso, violencia o intimidación al que se ve sometido.

- Las estrategias implementadas por los estudiantes como medidas de afrontamiento ante el acoso escolar son las centradas en la resolución y mediación de conflicto y las centradas en la evitación.

\section{Recomendaciones}

Autoridades del MINED:

- Capacitar a docentes en pedagogías para el manejo de ambientes interculturales.

- Ser más incluyentes de docentes pertenecientes a los grupos étnicos minoritarios.

- Implementar el Sistema Educativo Autonómico Regional en las escuelas. 
- Implementar programas de prevención de violencia o acoso dentro de los centros educativos mediante: Charlas alusivas a la temática acoso escolar. Establecer espacios que permita realizar actividades que se implemente el trabajo en equipo, la responsabilidad, formación de valores entre otras.

- Abordar abiertamente el tema de la Discriminación racial en las escuelas.

- Garantizar espacios de consejería dirigidos por profesionales de la salud mental.

- Capacitar a estudiantes en la aplicación de estrategias de afrontamiento asertivas para manejar situaciones de acoso escolar.

\section{A la dirección del centro escolar:}

- Dar a conocer a los profesores y autoridades del colegio sobre el "acoso escolar" como un problema sistémico que reclama solución a distintos niveles y además la importancia que se debe prestar a este peligro.

- Fomentar con ayuda del claustro de profesores y personal de los centros educativos, el respeto entre compañeros. Para que haya un clima escolar adecuado y respetuoso.

- Crear espacios de diálogos sobre la discriminación.

\section{A padres/madres de familia:}

- Fomentar en sus hijos respeto, comunicación asertiva, valores y responsabilidad.

- Involucrarse en la educación de sus hijos (Estado en contacto con el personal de la escuela y buscar información acerca de la forma en la que se relaciona su hijo con los otros compañeros).

- Conocer a los amigos de sus hijos y a la gente con quienes conviven a diario.

- En caso de experimentar violencia intrafamiliar, buscar ayuda profesional que les permita atender esta situación.

\section{A estudiantes:}

- Denunciar ante las autoridades escolares situaciones de acoso escolar.

\section{Lista de referencias}

Bandura, A. (1977). Social Learning Theory. General Learning Press.

Benítez, L. y Justicia, F. (2006). El maltrato entre iguales: Descripción y análisis del fenómeno. Revista Electrónica de Investigación Psicoeducativa, 9 (4), 151-170 http://www.sc.ehu.es/ptwgalam/meriales\%2odocentes\%2ocurso\%2overano\%2o 2008/1.\%20Bullying\%20aproximacion\%20al\%2ofenomeno\%20Onederra.pdf 


\section{EDUCACIÓN}

Hunter, S.; Mora, J. \& Ortega, R. (2004). The long-term effects of coping-strategy use in the victims of bullying. Spanish Journal of Psychology, 7, 3-12.

Kuntsche, E. (2006). Televisión viewing and forms of bullying among, Adolescents from eight countries. Recuperado de http.www.nytimes.com

Lazarus, R. s. y Folkman, S. (1984). Stress, Appraisal and Coping. NewYork: Springer Publication.

López, J (2000). Reporte de Acoso escolar, Bullying en las escuelas, intervención. Recuperado en: http://www.lacronica.com/EdicionEnLinea/Notas/ Noticias/o8102010/472483.aspx 10/ 2011.

Olweus D (1993). Bullying at school: What we know and what we can do. New York: Blackwell.

Olweus, D. (1978). Aggression in the schools: Bullies and whipping boys, Washington, DC: Hemisphere (Wiley).

Olweus, D. (1998). Conductas de acoso y amenazas entre escolares, Madrid, Ediciones Morata.

Oñederra, J. (2008). Bullying: concepto, Causas, Consecuencias, Teorías y Estudios Epidemiológicos.

Ortega, R. \& Del Rey, R. (2008). La violencia escolar en la Educación Primaria de Managua: comprender el contexto para abordar la realidad. Publicaciones, 39, 2009 pp. 31-41

Palomero, J. \& Fernández, M (2012). La Violencia Escolar: Un punto de vista global. Revista Interuniversitaria de formación de profesorado, 41, 19-38.

Rodríguez, D. F.; Gutiérrez, H.C.; Herrero, F. J.; Albuerna, F.; Cuesta, M.; Hernández E.; Gómez, C, P. (2004). Violencia en la enseñanza obligatoria: Alternativas desde la perspectiva del profesorado. Revista Electrónica de metodología aplicada. Vol. 9, Núm. 1, pp. 12-27 Recuperado en: http://www.unioviedo.net/reunido/index. php/Rema/article/view/9767

Rojas Marcos, L. (1995). Las semillas de la violencia.

Roth, Sy Cohen, L. J. (1986). Approach, avoidance, and coping with stress. Am. Psych $41,7,813-819$. 